OPEN ACCESS

Edited by:

Kailash C. Bansal,

ICAR-NBPGR, India

Reviewed by:

Narendra Singh Yadav,

Ben-Gurion University of the Negev,

Beersheba, Israel

Anil Kumar Singh,

ICAR-Indian Institute of Agricultural

Biotechnology, India

*Correspondence:

Guangyuan $\mathrm{He}$

hegy@hust.edu.cn

Guangxiao Yang

ygx@hust.edu.cn

Junli Chang

cjl@hust.edu.cn

${ }^{\dagger}$ These authors have contributed equally to this work.

Specialty section:

This article was submitted to

Plant Abiotic Stress,

a section of the journal

Frontiers in Plant Science

Received: 14 February 2017

Accepted: 24 July 2017

Published: 08 August 2017

Citation:

Wei Q, Luo Q, Wang R, Zhang F He Y, Zhang Y, Qiu D, Li K, Chang J, Yang $G$ and He G (2017) A Wheat R2R3-type MYB Transcription Factor TaODORANT1 Positively Regulates Drought and Salt Stress Responses

in Transgenic Tobacco Plants.

Front. Plant Sci. 8:1374.

doi: 10.3389/fpls.2017.01374

\title{
A Wheat R2R3-type MYB Transcription Factor TaODORANT1 Positively Regulates Drought and Salt Stress Responses in Transgenic Tobacco Plants
}

\section{Qiuhui Weit, Qingchen Luo', Ruibin Wang, Fan Zhang, Yuan He, Yang Zhang, Ding Qiu, Kexiu Li, Junli Chang*, Guangxiao Yang* and Guangyuan He*}

\begin{abstract}
The Genetic Engineering International Cooperation Base of Chinese Ministry of Science and Technology, Key Laboratory of Molecular Biophysics of Chinese Ministry of Education, College of Life Science and Technology, Huazhong University of

Science and Technology, Wuhan, China
\end{abstract}

MYB transcription factors play important roles in plant responses to biotic and abiotic stress. In this study, TaODORANT1, a R2R3-MYB gene, was cloned from wheat (Triticum aestivum L.). TaODORANT1 was localized in the nucleus and functioned as a transcriptional activator. TaODORANT1 was up-regulated in wheat under PEG6000, $\mathrm{NaCl}, \mathrm{ABA}$, and $\mathrm{H}_{2} \mathrm{O}_{2}$ treatments. TaODORANT1-overexpressing transgenic tobacco plants exhibited higher relative water content and lower water loss rate under drought stress, as well as lower $\mathrm{Na}^{+}$accumulation in leaves under salt stress. The transgenic plants showed higher CAT activity but lower ion leakage, $\mathrm{H}_{2} \mathrm{O}_{2}$ and malondialdehyde contents under drought and salt stresses. Besides, the transgenic plants also exhibited higher SOD activity under drought stress. Our results also revealed that TaODORANT1 overexpression up-regulated the expression of several ROS- and stress-related genes in response to both drought and salt stresses, thus enhancing transgenic tobacco plants tolerance. Our studies demonstrate that TaODORANT1 positively regulates plant tolerance to drought and salt stresses.

Keywords: wheat, abiotic stress, MYB, antioxidation system, stress related genes

\section{INTRODUCTION}

Plants are very often subjected to unfavorable environmental conditions, such as high salinity, drought, and extreme temperatures, which adversely affect plant growth, development, and productivity. To adapt to such environmental conditions, complex response mechanisms have been evolved in plants, including transcriptional regulation networks for the transduction of stress signals. The implementation of these intricate networks depends on the participation of various transcription factors (TFs), such as MYB, NAC, AP2/ERF, bZIP, bHLH, and WRKY (Qin et al., 2011; Chen et al., 2012).

The MYB superfamily plays a crucial role in abiotic stress responses. The first known MYB gene, isolated from maize (Zea mays) and designated COLORED1(C1), is required for anthocyanin synthesis in the aleurone of maize kernels (Paz-Ares et al., 1987). The MYB superfamily has been 
identified and analyzed in Arabidopsis and rice (Dubos et al., 2010; Katiyar et al., 2012). The MYB superfamily is divided into four subgroups based on the number of conserved MYB-domain repeats, i.e., a single or partial MYB repeat protein MYB1R, two repeats protein R2R3-MYB, three repeats protein MYB3R, and four R1/R2-like repeats protein 4R-MYB (Dubos et al., 2010). Several lines of evidence demonstrate that numerous R2R3-MYB proteins function in response to abiotic stress. In Arabidopsis, AtMYB96 overexpression enhances drought tolerance by integrating the ABA and auxin signaling pathways, while at the same time improving freezing and drought tolerance by regulating the target gene LTP3 (Seo et al., 2009; Guo et al., 2013). Meanwhile, AtMYB44 and AtMYB60 participate in plant responses to drought stress by regulating stomatal movement (Cominelli et al., 2005; Jung et al., 2008). AtMYB20-overexpressing Arabidopsis plants display salt-stress tolerance but susceptible to desiccation (Cui et al., 2013; Gao et al., 2014). AtMYB14 and AtMYB15 are known to participate in plant adaptation to freezing temperatures (Agarwal et al., 2006; Chen et al., 2013). In rice (Oryza sativa), the R2R3-type MYB gene OsMYB91 is involved in salt-stress tolerance and plant growth (Zhu et al., 2015). OsMYB2 has been implicated in tolerance to salt, freezing, and dehydration (Yang et al., 2012). In addition, MYB3R and MYB-related proteins, such as OsMYB511, OsMYB48-1, OsMYB4, and OsMYB3R-2, are known to be involved in stress adaptation (Dai et al., 2007; Pasquali et al., 2008; Xiong et al., 2014; Huang P. et al., 2015). GbMYB5 improves drought tolerance in transgenic cotton and tobacco plants (Chen et al., 2015). Overexpression of LeAN2, initially isolated from tomato plants, positively regulates responses to chilling and oxidative stresses in tobacco plants (Meng et al., 2014).

Given that wheat (Triticum aestivum L.) is a major grain crop, studies on the response of wheat to various adverse environments have attracted increasing attention. Significant progress in wheat genome sequencing has been achieved in recent years, laying the foundations for successful gene identification and isolation (Jia et al., 2013; Ling et al., 2013; Choulet et al., 2014; Mayer et al., 2014). Although many MYB genes have been identified in wheat, only a few have so far been characterized. Abiotic stress can induce TaMyb1 expression in the roots of wheat plants (Lee et al., 2006). Of the seven $M Y B$ gene fragments that were identified by Rahaie et al. (2010), TaMYBsdul was suggested to improve salt and drought tolerance in wheat (Rahaie et al., 2010). TaPIMP1 overexpression enhances disease, drought, and salt stress resistance in both transgenic tobacco and wheat (Liu et al., 2011; Zhang Z.Y. et al., 2012). Arabidopsis plants that overexpress TaMYB19 also show an improved tolerance to abiotic stress (Zhang et al., 2014).

In the present study, TaODORANT1, a R2R3-type MYB transcription factor gene, was cloned from wheat (T. aestivum cv. Chinese Spring). Gene expression profiles revealed that TaODORANT1 was up-regulated under high salinity, PEG6000, $\mathrm{H}_{2} \mathrm{O}_{2}$, and ABA treatments. TaODORANT1 overexpression conferred drought and salt tolerance to transgenic tobacco plants.

\section{MATERIALS AND METHODS}

\section{Plant Material and Treatments}

Wheat (T. aestivum cv. Chinese Spring) was used in this study. Seeds were germinated in the dark and were cultivated in a greenhouse $\left(12 \mathrm{~h} \mathrm{light} / 12 \mathrm{~h}\right.$ dark cycle at $\left.22^{\circ} \mathrm{C}\right)$. For the organ expression assay, roots, stems, and leaves were collected from 14-day-old seedlings. Mature roots, mature stems, mature leaves, stamens, and pistils were obtained from wheat plants at flowering stage. For drought and salt stress treatments, 14-day-old seedlings were cultured in solutions that contained 20\% PEG6000 (w/v) or $200 \mathrm{mM} \mathrm{NaCl}$. For $\mathrm{ABA}$ and hydrogen peroxide $\left(\mathrm{H}_{2} \mathrm{O}_{2}\right)$ treatments, seedling roots were dipped into $100 \mu \mathrm{M}$ ABA or $10 \mathrm{mM} \mathrm{H}_{2} \mathrm{O}_{2}$ solution, and the seedling leaves were sprayed with the same solutions. All samples were collected at the time points indicated, frozen in liquid nitrogen, and stored at $-80^{\circ} \mathrm{C}$ for subsequent RNA extraction.

\section{Cloning and Bioinformatic Analysis of TaODORANT1}

All known Arabidopsis and rice MYB protein sequences were acquired from relevant databases ${ }^{1,2}$. Expression profiles were predicted with PLEX $\mathrm{db}^{3}$. The accuracy and integrity of cDNA were verified with Ensembl Plants ${ }^{4}$ and IWGSC ${ }^{5}$. cDNA sequences were amplified with primer pairs (Supplementary Table S1) that were designed using the software Primer Premier 5 (PREMIER Biosoft, Palo Alto, CA, United States). Templates were synthesized from RNA mixtures that were extracted from wheat organs at different developmental stages and from seedlings that had been treated with $\mathrm{NaCl}$, PEG6000, ABA and $\mathrm{H}_{2} \mathrm{O}_{2}$. Polymerase chain reaction (PCR) products were sequenced (AuGCT Biotech, Beijing, China) with TaODORANT1 (accession no. KY013614) as the target gene. The TaODORANT1 promoter fragment was cloned via PCR with the primers listed in Supplementary Table S1. Promoter sequence was analyzed using the software PlantCARE 6 . Homologous TaODORANT1 protein sequences were collected from NCBI database ${ }^{7}$ and were aligned using the software ClustalX (Conway Institute, Dublin, Ireland). A phylogenetic tree was generated by using MEGA 5 software coupled with Neighbor-Joining method (Tamura et al., 2011).

\section{Subcellular Localization}

In order to detect the subcellular localization of TaODORANT1 in vivo, pMD18-T vector was constructed with the maize ubiquitin promoter and green fluorescent protein (GFP) gene to form the expression vector pMD18-ubi-GFP. Then, the open reading frame (ORF) of TaODORANT1 was amplified using specific primers that contained HindIII/SpeI restriction sites (Supplementary Table S1). The amplified TaODORANT1

\footnotetext{
${ }^{1}$ http://www.arabidopsis.org

${ }^{2}$ http://rice.plantbiology.msu.edu

${ }^{3}$ http://www.plexdb.org

${ }^{4}$ http://plants.ensembl.org/index.html

${ }^{5}$ http://www.wheatgenome.org

${ }^{6}$ http://bioinformatics.psb.ugent.be/webtools/plantcare/html

${ }^{7}$ https://blast.ncbi.nlm.nih.gov/Blast.cgi
} 
ORF was fused to the $5^{\prime}$-terminal end of the GFP gene in the pMD18-ubi-GFP vector to generate a recombinant vector. The recombinant vector ubiqutin::TaODORANT1-GFP and the control vector pMD18-Ubi-GFP were transformed into onion epidermal cells, respectively, via particle bombardment. The results were observed with fluorescence microscopy (IX71, Olympus, Japan).

\section{Transactivational and Binding Activity Analysis}

Transcriptional activity was investigated using the Clontech Matchmaker $^{\text {TM }}$ Yeast One-Hybrid system (TBUSA, Mountain View, CA, United States), a GAL4-based yeast one-hybrid system. The ORF at 1-795 base pair (bp) as well as various truncated ORFs at 1-360, 186-795, 361-795, and 513-795 bp of TaODORANT1 were amplified by PCR using specific primers that contained EcoRI/BamHI restriction sites (Supplementary Table S1). These fragments were then inserted into the pGBKT7 vector. An empty pGBKT7 vector was used as the negative control plasmid. For the binding activity assay, TaODORANT1 and three typical MYB binding motifs were introduced into the pGADT7 and pHIS2 plasmids (Supplementary Table S1). The recombinants were co-transformed into the yeast strain Y187. Yeast transformation and screening were performed in accordance with the users' manual (Clontech, United States).

\section{Expression Analysis of TaODORANT1 in Wheat}

Total RNA was extracted from different samples with a Plant Total RNA Extraction Kit (Zoman, Beijing, China). First-strand cDNA was synthesized with the FastQuant RT Kit (TIANGEN, Beijing, China). Quantitative real-time PCR (qRT-PCR) was performed with SuperReal PreMix Plus Kits (TIANGEN, Beijing, China). Each reaction solution contained $5 \mu$ l of $2 \times$ SuperReal PreMix, $1 \mu \mathrm{l}$ of gene-specific primers, and $1 \mu \mathrm{l}$ of cDNA (about $50 \mathrm{ng}$ ), and was added with $\mathrm{ddH}_{2} \mathrm{O}$ to a final volume of $10 \mu \mathrm{l}$. The PCR program was as follows: $95^{\circ} \mathrm{C}$ for $15 \mathrm{~min} ; 50$ cycles of $95^{\circ} \mathrm{C}$ for $10 \mathrm{~s} ; 55^{\circ} \mathrm{C}$ for $20 \mathrm{~s} ; 72^{\circ} \mathrm{C}$ for $30 \mathrm{~s}$. qRT-PCR was performed with the CFX Connect Real-Time System (Bio-Rad, Hercules, CA, United States). Expression data were analyzed with the comparative $2^{-\Delta \Delta C_{\mathrm{T}}}$ method (Livak and Schmittgen, 2001). The primers used in this assay are listed in Supplementary Table S1. The housekeeping wheat gene actin (accession no. AB181991.1) was used as the internal control.

\section{Plant Transformation}

To generate transgenic tobacco plants that overexpressed TaODORANT1, the ORF that contained the terminator codon was cloned into the pBI121 vector under the control of the cauliflower mosaic virus $35 \mathrm{~S}$ promoter with $\mathrm{XbaI} / \mathrm{BamHI}$ restriction sites. The pBI121-TaODORANT1-GFP constructs and pBI121-GFP vector were transformed into Agrobacterium tumefaciens strain EHA105. Transformation was accomplished using the A. tumefaciens-mediated leaf disk method (Horsch et al., 1985). Eight independent transgenic $\mathrm{T}_{2}$ lines were obtained.
The expression level of TaODORANT1 in each line was examined by RT-PCR.

\section{Southern Blotting Analysis}

Genomic DNAs of wildtype (WT) and transgenic tobacco plants were extracted by CTAB method (Staccy and Isaac, 1994), and were digested by restriction enzyme HindIII. Then, the digested gDNAs were separated by electrophoresis and transferred to Hybond-N+ membrane according to the manufacturer's protocol (Roche). Membrane was hybridized with digoxigenin (DIG) labeled probe (TaODORANT1). Finally, hybridized probe DNA was observed by exposure to Kodak double-emulsion films. The probe primers were listed in Supplementary Table S1.

\section{Stress Tolerance Analysis of the Transgenic Plants}

Wildtype, vacant vector control (VC), and overexpression (OE) lines were used to analyze stress tolerance. Seeds were surfacesterilized with $75 \%$ ethanol for $1 \mathrm{~min}$ and $10 \% \mathrm{H}_{2} \mathrm{O}_{2}$ for $8 \mathrm{~min}$. The seeds were then sown on 1/2 Murashige and Skoog (MS) medium and incubated in a growth chamber $(16 \mathrm{~h}$ light $/ 8 \mathrm{~h}$ dark cycle at $22^{\circ} \mathrm{C}$ ) for 10 days. The seedlings were transferred to $1 / 2 \mathrm{MS}$ media that contained $150 / 300 \mathrm{mM}$ mannitol or $150 / 200 \mathrm{mM} \mathrm{NaCl}$ for osmotic and salt stress assays. Root length was measured after 10 days of treatment. To analyze the stress tolerance of transgenic plants, 2-week-old seedlings were planted in pots that were filled with an equal quantity of moisture and soil and grown in a greenhouse under a $12 \mathrm{~h}$ light $/ 12 \mathrm{~h}$ dark cycle at $22^{\circ} \mathrm{C}$. In each biological replicate, about 50 tobacco plants (10 pots) of each line were treated. For the drought stress tolerance assay of transgenic plants, 4-week-old plants were withheld water for 27 days and then re-watered for 1 week. For the salt stress tolerance assay of transgenic plants, 3-week-old plants grown in pots were treated in a container containing $2 \mathrm{~L} 500 \mathrm{mM} \mathrm{NaCl}$ for 19 days. Supplemental $\mathrm{NaCl}$ solution was added to the container every 3 days throughout the treatment period. The water loss assay was performed as described by Huang Q. et al. (2015). The stomatal aperture assay was accomplished in accordance with Yan et al. (2014) with slight modifications: the duration of dehydration and ABA $(50 \mu \mathrm{M})$ treatment were modified to $40 \mathrm{~min}$ and $1 \mathrm{~h}$, respectively.

\section{Measurement of RWC, IL, MDA, and $\mathrm{H}_{2} \mathrm{O}_{2}$ Contents and CAT, SOD, and POD Enzyme Activities}

Leaves (second or third leaves from the top) with similar size were sampled from the WT, VC, and OE plants that were exposed to drought and salt stress. Relative water content (RWC) and ion leakage (IL) were determined as described by $\mathrm{Hu}$ et al. (2013). Malondialdehyde (MDA) accumulation was measured using the thiobarbituric acid-based method with an MDA assay kit (Jiancheng, Nanjing, China). $\mathrm{H}_{2} \mathrm{O}_{2}$ content as well as CAT, SOD, and POD activities were measured by spectrophotometry using the corresponding assay kits (Jiancheng, Nanjing, China). Superoxide anion radicals $\left(\mathrm{O}_{2}{ }^{-}\right)$and $\mathrm{H}_{2} \mathrm{O}_{2}$ were histochemically 
detected with the DAB and NBT staining method in accordance with the procedure by Hu et al. (2013).

\section{Measurement of Chlorophyll Content and $\mathrm{Na}^{+}$and $\mathrm{K}^{+}$Concentrations}

Chlorophyll content was measured as described by Arnon (1949). $\mathrm{Na}^{+}$and $\mathrm{K}^{+}$concentrations were detected with plant total $\mathrm{Na}^{+}$ and $\mathrm{K}^{+}$kits (Keming, Suzhou, China) in accordance with the kits' protocols. Leaves were washed with Milli-Q water, dried at $70^{\circ} \mathrm{C}$, and then ground to power. The powdered samples were used for ion concentration measurement. To measure $\mathrm{Na}^{+}$concentration, $\mathrm{Na}^{+}$was reacted with potassium pyroantimonate to form sodium pyroantimonate sediment under special conditions. To measure $\mathrm{K}^{+}$concentration, $\mathrm{K}^{+}$was reacted with sodium tetraphenylborate to form potassium tetraphenylborate sediment under special conditions. Then, the turbidity of the test solution was measured at $520 \mathrm{~nm}$ using a spectrophotometer.

\section{Expression Assay of Stress-Related Genes}

Two-week-old tobacco seedlings were treated on 1/2 MS media with $300 \mathrm{mM}$ mannitol or $150 \mathrm{mM} \mathrm{NaCl}$ for 1 week. Then the total RNA of the seedlings was extracted to analyze the expression of stress-induced genes by qRT-PCR. The specific primers used are listed in Supplementary Table S1. The Ntubiqutin gene was selected as the internal control.

\section{Statistical Analysis}

Statistical analysis was performed with SPSS (IBM Analytics, New York, NY, United States) and Student's $t$-test.

\section{RESULTS}

\section{Identification of TaODORANT1}

Wheat MYB expressed sequence tags (ESTs) were obtained by searching the NCBI UniGene database ${ }^{8}$ with known Arabidopsis and rice MYB sequences listed in Supplementary Table S3. The obtained ESTs were assembled into longer cDNA sequences. A cDNA sequence was selected based on the data acquired from an expression database ${ }^{3}$ (Supplementary Figure S3). The accuracy and integrity of this cDNA sequence were confirmed by Ensembl Plants ${ }^{4}$ and IWGSC ${ }^{5}$. The putative $M Y B$ gene was identified and cloned from wheat. The cloned cDNA sequence is 1,039-bp long containing a 798-bp ORF, which was predicted to encode a 265 amino acid protein with a relative molecular mass of $29.211 \mathrm{kDa}$ and an isoelectric point of 6.17. A phylogenetic tree was generated with this MYB sequence and its orthologs from different plant species. Phylogenetic analysis showed that the MYB sequence had the closest relationship with protein ODORANT1 from Aegilops tauschii (Figure 1A). Therefore, the putative MYB gene was designated TaODORANT1. Aligning TaODORANT1 with homologous proteins from other plant species revealed two conversed repeats in the DNA-binding

${ }^{8}$ https://www.ncbi.nlm.nih.gov/unigene domain, which classified TaODORANT1 to the R2R3-type MYB subfamily (Figure 1B).

\section{Subcellular Localization and Transactivation Activity Analysis of TaODORANT1}

To confirm the localization of TaODORANT1 in vivo, a vector that expressed the fused TaODORANT1-GFP protein under the control of a maize ubiquitin promoter was constructed. Transient expression of the fused protein gene in onion epidermal cells showed that the fluorescence of TaODORANT1-GFP was exclusively localized in the nucleus, whereas that of the control GFP protein was diffused throughout the cell (Figure 2A). These results suggested that TaODORANT1 was a nuclear-localized protein.

The transactivation activity of TaODORANT1 was verified with a transactivation assay. To construct GAL4BD-TaODORANT1 recombinant, complete and various truncated TaODORANT1 ORFs were cloned into pGBKT7 plasmids. The recombinants were transformed into the yeast strain AH109 to examine the transactivation ability of TaODORANT1. All transformants, including the negative control pGBKT7, grew well on the SD/-Trp medium, while just transformants containing the TaODORANT1 C-terminal grew well and turned blue on SD-Trp/His and SD-Trp/His/Ade medium with $\mathrm{x}$ - $\alpha$-galactoside ( $\mathrm{x}$ - $\alpha$-gal) (Figure 2B). These results indicated that TaODORANT1 had transactivation activity, which can be attributed to the amino acid residues at C-terminal 171 to 265. Furthermore, binding activity analysis showed that the fused protein pGADT7-TaODORANT1 was able to bind to type II but not to type I and IIG MYB binding motifs (Figure 2C).

\section{Expression Pattern Analysis of TaODORANT1 in Wheat}

qRT-PCR was used to examine TaODORANT1 expression in various wheat organs at different developmental stages. Results showed that TaODORANT1 was expressed in all examined organs. The highest transcript levels were observed in the roots of 2-week-old seedlings. The lowest expression levels were observed in mature stems (Figure 3A). To gain insight into the function of TaODORANT1, its expression levels were measured under various stress treatments. TaODORANT1 expression rapidly increased to 6.1 -fold at $1 \mathrm{~h}$, and then gradually returned to normal levels after $3 \mathrm{~h}$ to $12 \mathrm{~h}$ of $20 \%$ PEG6000 treatment. After $24 \mathrm{~h}$ of $20 \%$ PEG6000 treatment, TaODORANT1 expression sharply increased to its highest level of approximately nine-fold (Figure 3B). After treatment with $200 \mathrm{mM} \mathrm{NaCl}, \mathrm{TaODORANT1}$ expression increased 2.8 -fold at $1 \mathrm{~h}$ and then quickly decreased (Figure 3C). Given that $\mathrm{ABA}$ and $\mathrm{H}_{2} \mathrm{O}_{2}$ are induced and accumulate as signal molecules under drought and salt stresses, TaODORANT1 expression levels were also examined after ABA and $\mathrm{H}_{2} \mathrm{O}_{2}$ treatments. Under $100 \mu \mathrm{M}$ ABA treatment, TaODORANT1 expression levels initially increased, gradually reached peak, and then decreased (Figure 3D). Under $10 \mathrm{mM}$ $\mathrm{H}_{2} \mathrm{O}_{2}$ treatment, TaODORANT1 expression increased (6.8-fold) at $1 \mathrm{~h}$ and then gradually decreased to a normal level (Figure 3E). 
A

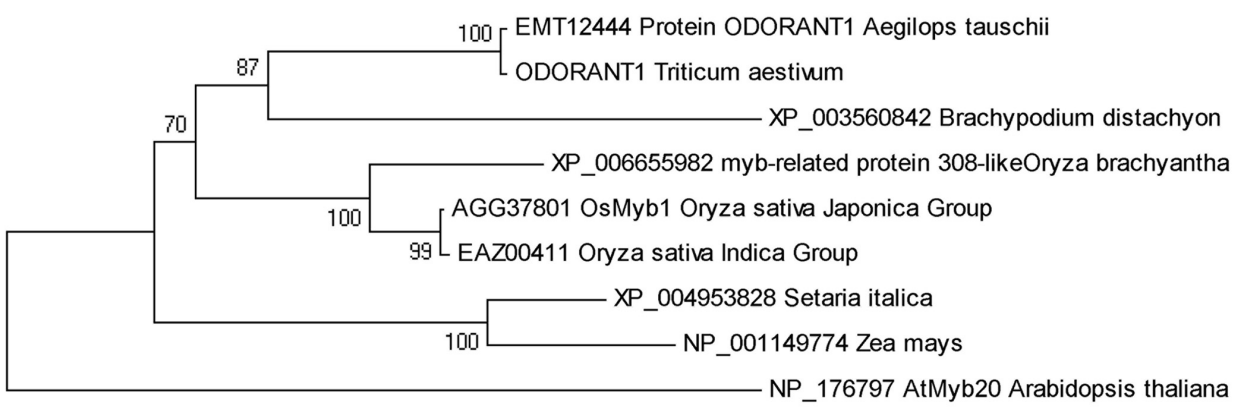

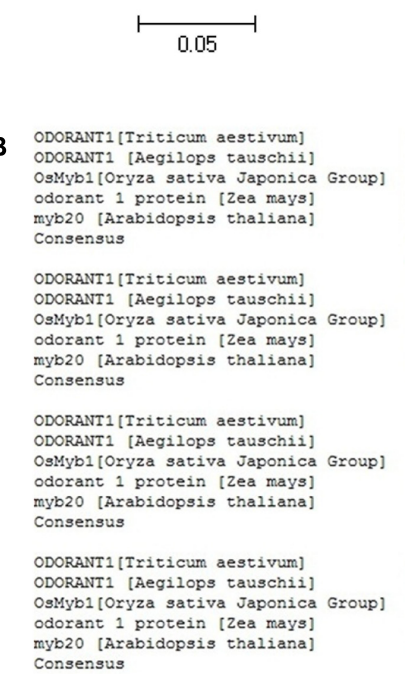
ODORANT1[Triticum aestivum] ODMANT1 [Aegrlops tauschil Group odorant 1 protein [Zea mays] (a) I pativa Japonica] MMyb1 [Oryza gativa Japonica Group] Consensus
Titriticum aestivum] 1 protein [zea mays] Consensus

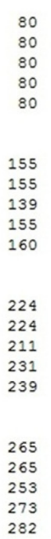

FIGURE 1 | Sequence and phylogenetic tree analysis of TaODORANT1. (A) Phylogenetic relationship of TaODORANT1 with its orthologs from other plant species. (B) Sequence alignment of TaODORANT1 and homologous proteins from other plant species. The black background represents identical amino acid residues in the aligned sequences. Red straight lines indicate the conserved domains of R2R3-MYB.

These results demonstrated that the expression of TaODORANT1 in wheat was induced by PEG6000, $\mathrm{NaCl}, \mathrm{H}_{2} \mathrm{O}_{2}$, and ABA. To further understand the regulatory mechanism of TaODORANT1 expression, a 1,800-bp fragment in the promoter region of TaODORANT1 was cloned and analyzed. Many abiotic stress response elements (MBS, LTR, HSE, ARBE, and ERE) were found in the promoter sequence (Supplementary Table S2). Based on these results, we speculated that TaODORANT1 plays a key role in plant response to adverse environments.

\section{Ectopic Overexpression of TaODORANT1 Enhances Drought and Salt Tolerance in Transgenic Tobacco Seedlings}

To further investigate the function of TaODORANT1 in abiotic stress tolerance, transgenic tobacco plants were generated. Then, the expression levels of transgene were detected by semi-qRT-PCR, and result showed that the three TaODORANT1-overexpressing lines OE1, OE3, and OE12 had higher expression levels. The copy number of transgene was detected by Southern blotting, and the result revealed that one (OE3) or two copies (OE1 and OE12) of TaODORANT1 were integrated into the genome of these three tobacco plants
(Supplementary Figure S1). Therefore, these three independent lines were selected to analyze the function of TaODORANT1 in our study. Under normal conditions, the WT, VC, and OE lines showed similar phenotypes. For the drought/salt stress tolerance assay of TaODORANT1 overexpressing plants, the 10-day-old post-germination seedlings were vertically grown on $1 / 2 \mathrm{MS}$ media that contained $150 / 300 \mathrm{mM}$ mannitol or $150 / 200 \mathrm{mM}$ $\mathrm{NaCl}$ for 10 days. The transgenic and control lines showed no difference in growth on 1/2 MS plates. By contrast, the growth of the control lines on stress media was more inhibited than that of the OE lines (Figures 4A-E). Statistical analysis of root length also showed that the overexpression lines grew better than the control lines under $\mathrm{NaCl}$ and mannitol treatments (Figure 4F). These results indicated that TaODORANT1 overexpression enhanced tobacco seedlings tolerance to drought and salt stresses.

\section{Drought Tolerance Assay of TaODORANT1 Overexpression Tobacco Plants}

To further investigate drought tolerance of TaODORANT1 overexpressing plants, water was withheld from 4-week-old 
A

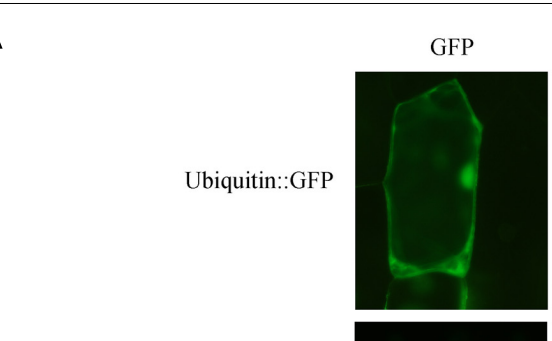

Ubiquitin::TaODORANT1-GFP
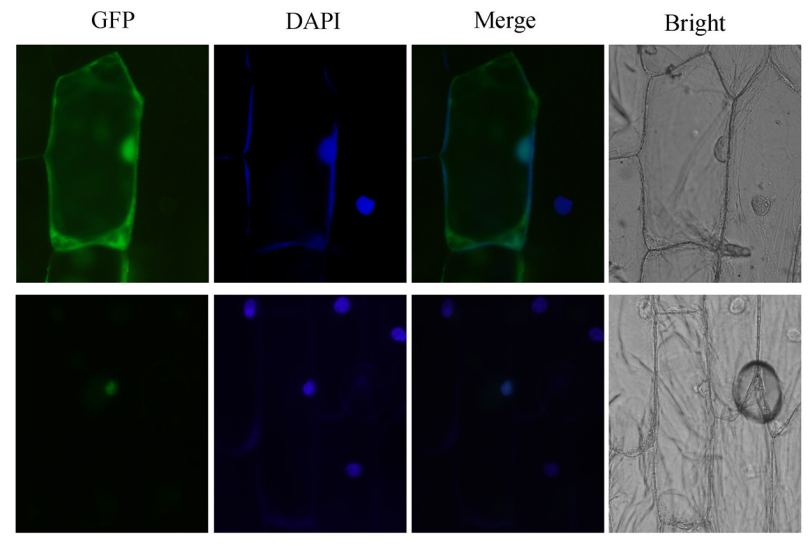

SD-T SD-T/H+x- $\alpha$-gal SD-T/H/A+x- $\alpha$-gal

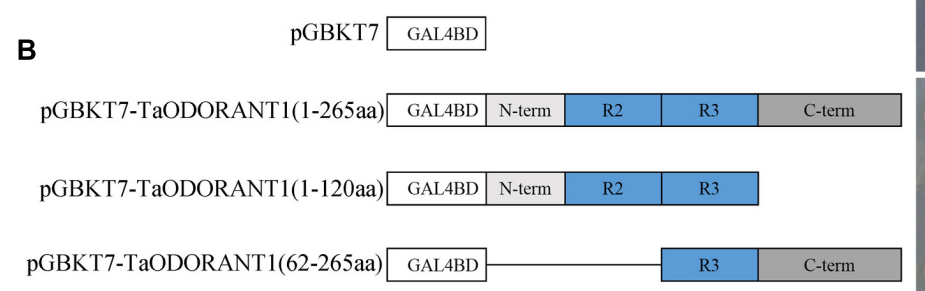

pGBKT7-TaODORANT1(121-265aa) GAL4BD C-term

\begin{tabular}{lll} 
pGBKT7-TaODORANT1(171-265aa) GAL4BD & C-term \\
\hline
\end{tabular}
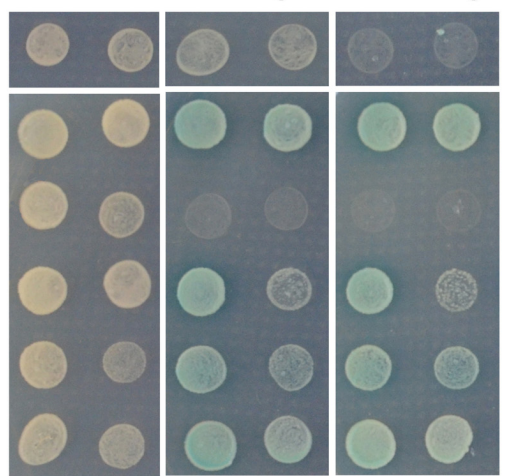

\section{.}

Dilution

pGADT7-TaODORANT1/pHIS2

pGADT7-TaODORANT1/pHIS2-motifl

pGADT7-TaODORANT1/pHIS2-motifII

pGADT7-TaODORANT1/pHIS2-motiflIG

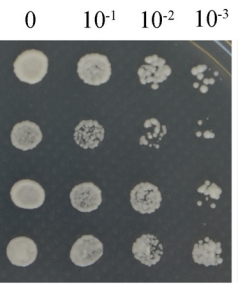

$\mathrm{SD}-\mathrm{T} / \mathrm{L} / \mathrm{H}$
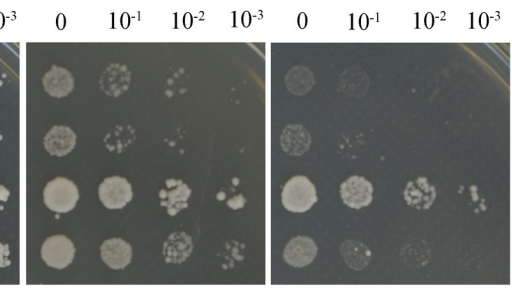

SD-T/L/H+20mM 3-AT SD-T/L/H+40mM 3-AT SD-T/L/H+60mM 3-AT

FIGURE 2 | Subcellular localization and transcriptional activity analysis of TaODORANT1. (A) Subcellular localization of TaODORANT1. Recombinant vector ubiqutin::TaODORANT1-GFP and control vector ubiquitin::GFP were transformed into onion epidermal cells and observed with fluorescence microscopy, respectively. (B) Transactivation activity of TaODORANT1 in yeast. Schematic diagrams illustrate the different portions of TaODORANT1 ORF that were introduced into the pGBKT7. Recombined vectors were transformed into yeast strain AH109, and the transformants were screened by SD/-Trp, SD/-Trp/-His+X- $\alpha$-gal, $\mathrm{SD} /-\mathrm{Tr}$ /-His/-Ade+X- $\alpha$-gal media. (C) Binding activity assay of TaODORANT1. Three MYB binding motifs I, II, and IIG were fused with pHIS2 vector, and the TaODORANT1 ORF was fused with pGADT7 vector. Reconstructed pGADT7 and pHIS2 vectors were co-transformed into yeast strain Y187. Transformants were incubated on SD/-Trp/-Leu/-His media with different concentrations of 3-AT. Three independent biological replicates were performed and produced similar results.

plants in soil for 27 days. The WT and VC lines became seriously chlorotic at an early stage, wilted, and even died. By contrast, the transgenic lines OE1, OE3, and OE12 showed only chlorosis and few deaths (Figure 5A). After re-watering for 1 week, the survival rate of the transgenic plants was $60-80 \%$, which was obviously higher than those of WT and VC $(<20 \%)$ (Figure 5B). The RWC of leaves was measured as an important parameter of drought tolerance. The OE1 and OE12 lines exhibited higher RWC (70-80\%) than that of the control plants (40-45\%) (Figure 5C). Consistent with the drought tolerance phenotype, the water loss rate of detached leaves from the $\mathrm{WT}$ and $\mathrm{VC}$ lines was also higher than those from transgenic lines (Figure 5D). Moreover, the WT leaves presented serious coiling after $24 \mathrm{~h}$ of dehydration (Figure 5E). As more than $95 \%$ of water loss in plants is via transpiration (Schroeder et al., 2001), the status of stomatal closure was observed and the stomatal width:length ratio was measured under dehydration or exogenous ABA treatments. Result showed that the stomatal aperture of OE1 was smaller than that of the WT (Figures 5F,G). These results demonstrated that TaODORANT1 positively regulated drought stress response. 
A

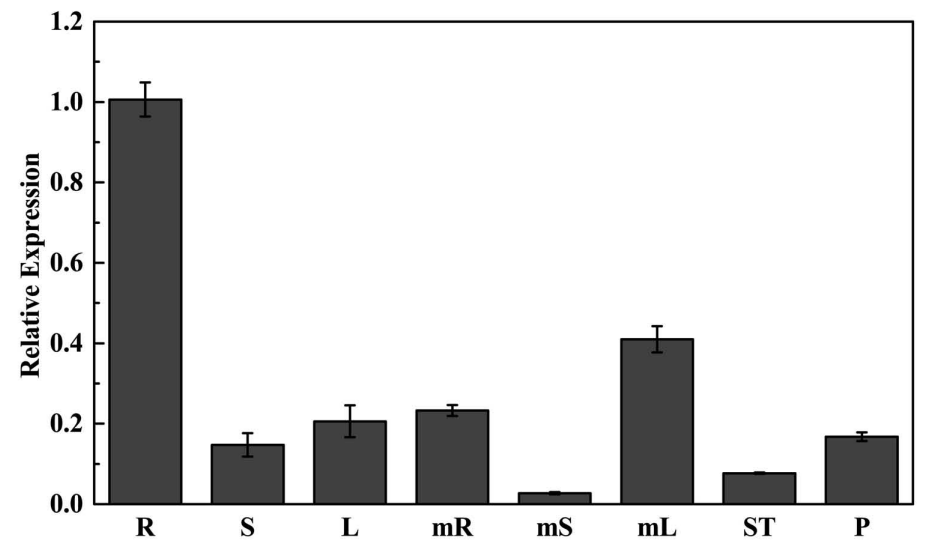

B

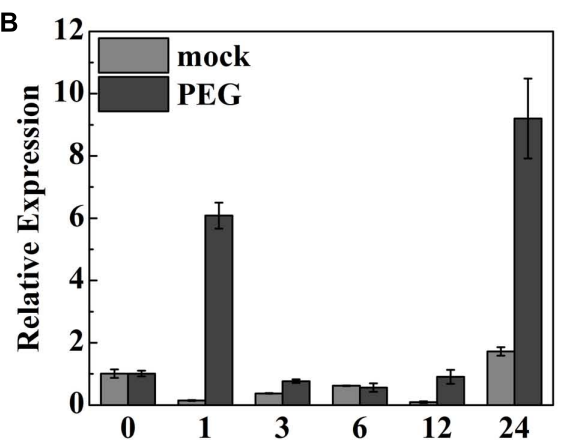

Hours post PEG treatment(h)

D

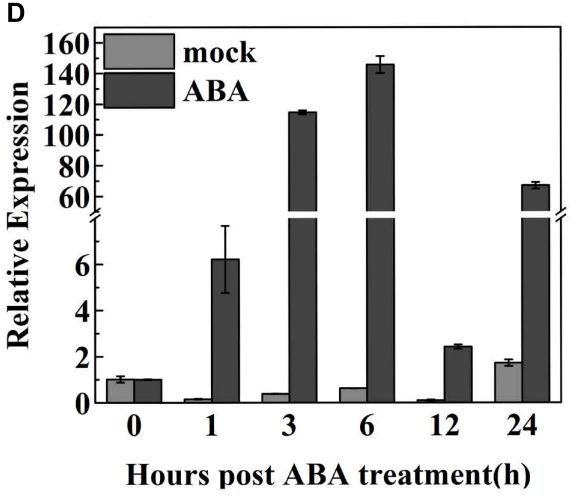

C

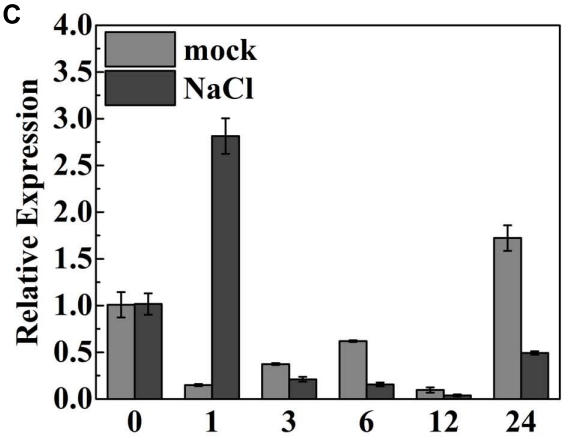

Hours post $\mathrm{NaCl}$ treatment(h)

E

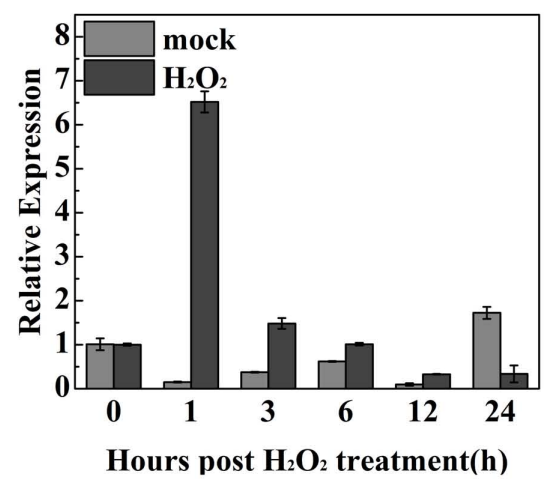

FIGURE 3 | Expression profiles of TaODORANT1 in wheat. (A) Organ-specific expression assay of TaODORANT1 in wheat (R: seedlings root; S: seedlings stem; L: seedlings leaf; $\mathrm{mR}$ : mature root; $\mathrm{mS}$ : mature stem; $\mathrm{mL}$ : mature leaf; ST: stamen; P: pistil). (B-E) Expression patterns of TaODORANT1 in 14-day-old wheat seedlings after treatment with 20\% PEG6000, $200 \mathrm{mM} \mathrm{NaCl}, 100 \mu \mathrm{M} \mathrm{ABA}$, and $10 \mathrm{mM} \mathrm{H}_{2} \mathrm{O}_{2}$, respectively. Three independent biological replicates were performed and produced similar results. Vertical bars refer to \pm SE $(n=3)$.

\section{Salt Stress Tolerance Assay of TaODORANT1-Overexpressing Tobacco Plants}

To analyze the salt stress tolerance of TaODORANT1 overexpressing plants, 3-week-old plants were irrigated with $500 \mathrm{mM} \mathrm{NaCl}$ solution for 19 days. The WT and VC lines exhibited serious chlorosis and death, whereas the transgenic plants remained green (Figure 6A) and showed a higher survival rate (50-60\%) than WT and VC (Figure 6B). The reduced chlorophyll content of the leaves of WT and VC plants also reflected this observed phenotype (Figure 6C). To further understand the mechanism underlying the enhanced salt tolerance of TaODORANT1 overexpressing plants, $\mathrm{Na}^{+}$and $\mathrm{K}^{+}$accumulation in leaves was detected after salt stress. No significant difference of $\mathrm{K}^{+}$concentration was observed between WT and $\mathrm{OE}$ lines (Figure 6E). However, $\mathrm{Na}^{+}$concentration was significantly lower in OE lines than WT (Figure 6D). Therefore, the $\mathrm{K}^{+} / \mathrm{Na}^{+}$ratios in $\mathrm{OE}$ lines were higher than that in WT after salt stress (Figure 6F). The expression levels of two 


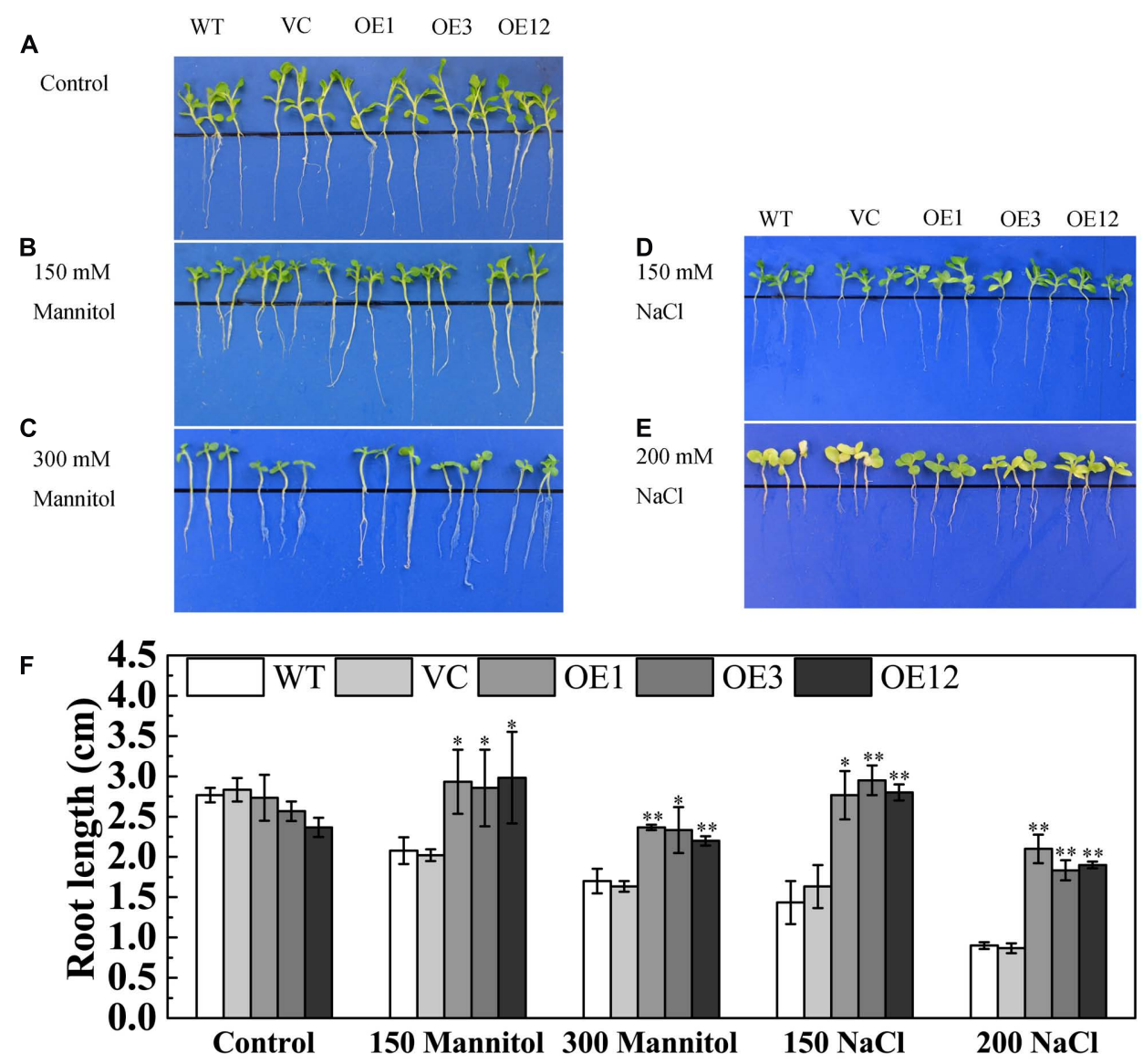

FIGURE 4 | Tolerance analysis of 10-day-old tobacco seedlings. (A-E) Phenotype of seedlings after treatment with mannitol or NaCl for 10 days. (F) Root length statistics of seedlings after treatment with mannitol or $\mathrm{NaCl}$ for 10 days. Three independent biological replicates were performed and produced similar results. Vertical bars refer to $\pm \mathrm{SE}(n=3)$. Asterisks indicate significant difference between WT and transgenic lines $\left({ }^{*} P<0.05 ; * * P<0.01\right)$.

representative ion transporter genes were examined by qRT-PCR. The expression levels of NtSOS1 and NtNHX2 were higher in transgenic plants (OE3 and OE12) than those in WT plants (Figures 6G,H). These results suggested that TaODORANT1 regulates the expression of $\mathrm{Na}^{+}$and $\mathrm{K}^{+}$transporter genes, thereby reducing $\mathrm{Na}^{+}$accumulation in photosynthetic tissue and improving salt stress tolerance of transgenic plants.

\section{Oxidative Damage Assay of TaODORANT1 Overexpression Tobacco Plants under Drought and Salt Stresses}

Drought and salt stresses disrupt cellular homeostasis and increase the accumulation of reactive oxygen species (ROS), especially $\mathrm{O}_{2}{ }^{-}$and $\mathrm{H}_{2} \mathrm{O}_{2}$ (Polle, 2001). The histochemical detection of $\mathrm{H}_{2} \mathrm{O}_{2}$ and $\mathrm{O}_{2}{ }^{-}$in leaves was accomplished by DAB and NBT staining. Under normal conditions, $\mathrm{H}_{2} \mathrm{O}_{2}$ and $\mathrm{O}_{2}{ }^{-}$ contents were relatively lower and not significantly different between WT and OE1 lines. After drought or $\mathrm{NaCl}$ treatment, the staining of WT and OE1 leaves was deeper than that seen in the control. However, the staining of OE1 leaves was lighter than the WT (Figure 7A), which indicated that transgenic plants accumulated less $\mathrm{H}_{2} \mathrm{O}_{2}$ and $\mathrm{O}_{2}^{-}$. Under normal conditions, IL and MDA content in the leaves of the WT, VC, and $\mathrm{OE}$ lines were not significantly different. After drought stress, the accumulation of MDA in OE3 was significantly less than WT and VC. After salt stress, the accumulation of MDA in transgenic plants (OE1, OE3, and OE12) was significantly lower when compared with the WT and VC. Moreover, the WT and VC leaves presented severe IL compared with transgenic plants after drought and salt stresses (Figures 7B,C). These results indicated that TaODORANT1 overexpression decreased the oxidative damage caused by drought and salt stresses.

\section{Overexpression of TaODORANT1 Regulates the Expression Levels of Antioxidation-Related Genes and Improves the Activity of Antioxidative Enzymes under Drought and Salt Stresses}

An efficient antioxidation system, that alleviates oxidative damage, is required to enhance drought and salt tolerance 


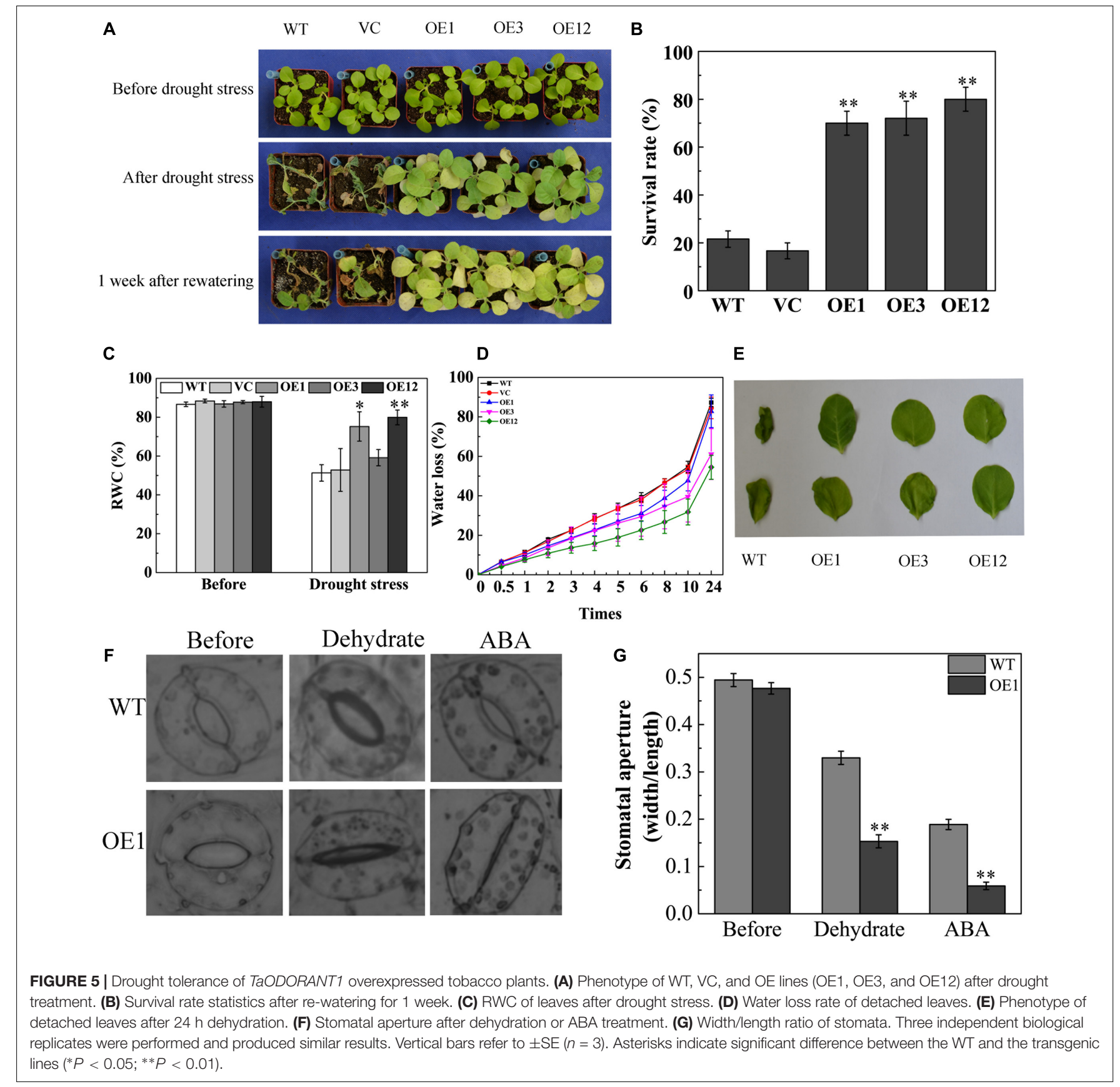

(Bartels and Sunkar, 2005). The expression levels of CAT, SOD, and $P O D$ genes, related to ROS-scavenging enzymes, were examined by qRT-PCR. After drought stress, the transcription levels of CAT, $S O D$, and POD were higher in $O E$ lines than those in WT (Figures 8A-C). On the other hand, expression of the respiratory burst oxidase homolog $(R b o h F)$, a ROS producer, significantly decreased in the OE lines (Figure 8D). $\mathrm{H}_{2} \mathrm{O}_{2}$ content and antioxidative enzyme activities in leaves were detected after drought stress. The results showed that $\mathrm{OE}$ lines exhibited higher activities of CAT and SOD, and less $\mathrm{H}_{2} \mathrm{O}_{2}$ accumulation than the WT and VC after drought stress (Figures 8E-G). However,
POD activity was not significantly different between the $\mathrm{OE}$ lines and the control (Figure 8H). After salt treatment, the CAT transcript levels were higher in the OE3 and OE12 lines than those in the WT (Figure 8I). Enhanced CAT activity, which directly decomposes $\mathrm{H}_{2} \mathrm{O}_{2}$, reduced accumulation of $\mathrm{H}_{2} \mathrm{O}_{2}$ in transgenic plants (Figures 8J,K). However, SOD and POD activities were not significantly different between the OE lines and the control (Supplementary Figure S2). These results showed that TaODORANT1 enhanced ROS-scavenging ability by activating the activities of different enzymes in response to drought and salt stresses. 
A
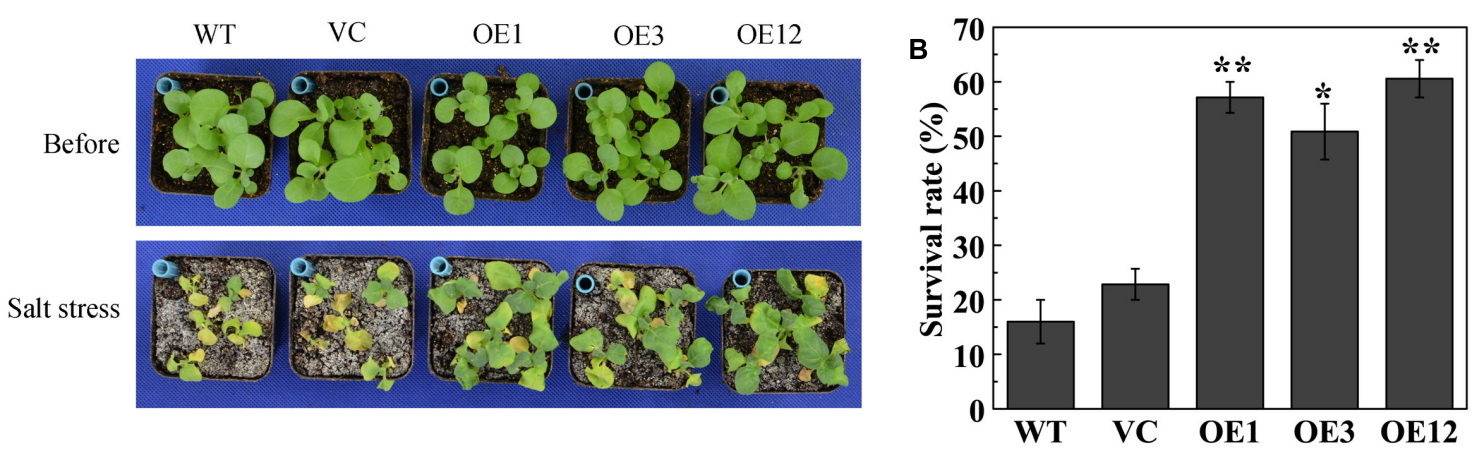

C
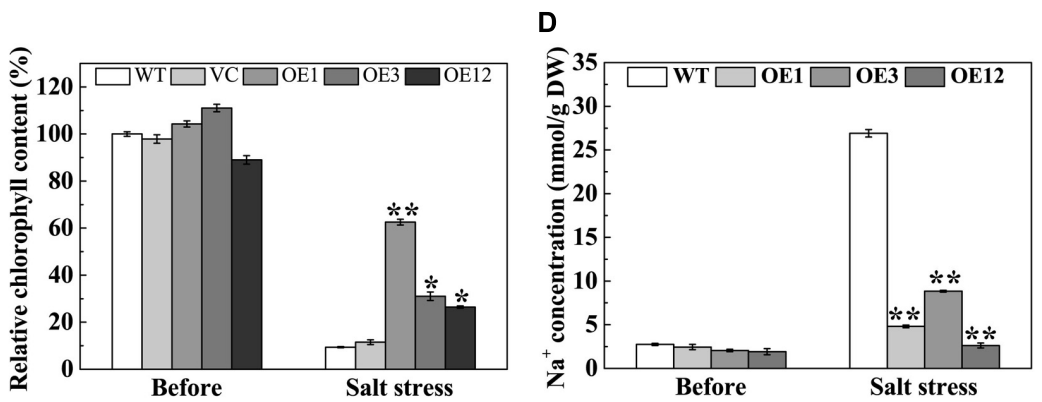

E
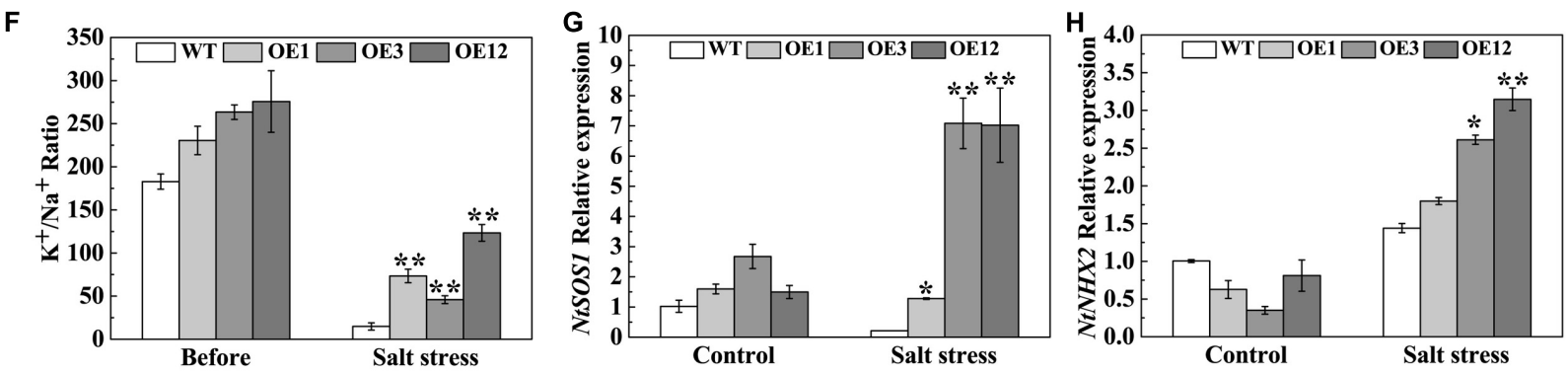

FIGURE 6 | Salt tolerance of TaODORANT1 overexpressed tobacco plants. (A) Phenotype of WT, VC and OE lines (OE1, OE3, and OE12) after salt treatment. (B) Survival rate statistical analysis. (C) Chlorophyll content in leaves. (D,E) $\mathrm{Na}^{+}$and $\mathrm{K}^{+}$concentrations in leaves. (F) $\mathrm{K}^{+} / \mathrm{Na}^{+}$ratio. (G,H) Expression levels of ion transporter genes. Three independent biological replicates were performed and produced similar results. Vertical bars refer to \pm SE $(n=3)$. Asterisks indicate significant difference between the WT and the transgenic lines $\left({ }^{*} P<0.05\right.$; $\left.{ }^{* *} P<0.01\right)$.

\section{TaODORANT1 Regulates the Expressions of Stress-Related Genes under Drought and Salt Stresses}

To further understand the functional mechanisms of TaODORANT1 at the molecular level, qRT-PCR was used to examine the levels of stress-related genes, including NtNCED3 (9-cis-epoxycarotenoid dioxygenase), NtERD10C/D (early responsive to dehydration), NtABF2 (ABA-responsive element binding), NtLEA5 (late embryogenesis-abundant protein), TobLTP1 (lipid-transfer protein), NtP5CS1 ( $\triangle 1$-pyrroline-5-carhoxylate synthetase 1), NtSAMDC (S-adenosyl-L-methionine decarboxylase), and NtADC (arginine decarboxylase). Under $300 \mathrm{mM}$ mannitol or $150 \mathrm{mM} \mathrm{NaCl}$ treatment, the expression levels of NtNCED3, NtERD10C, NtERD10D, NtLEA5, NtABF2, TobLTP1, NtP5CS1, NtSAMDC, and $N t A D C$ were higher in transgenic lines (OE3 and OE12) than those in WT. However, the expression of some related genes in OE1 were not significantly induced after drought and salt stresses (Figure 9). These results suggested that TaODORANT1 overexpression in tobacco plants enhanced the drought and salt tolerance by regulating the expression of stress-related genes.

\section{DISCUSSION}

Biotic and abiotic stresses seriously influence the growth, development, and productivity of crop plants. Therefore, stress tolerance genes should be explored for the genetic improvement of crops. Although many TFs involved in plants abiotic stress have been identified and characterized, few MYB genes have so far been characterized in wheat. Furthermore, the mechanisms through which $M Y B$ genes enhance plant drought and salt stress tolerance remain unclear. In our study, a R2R3-type 


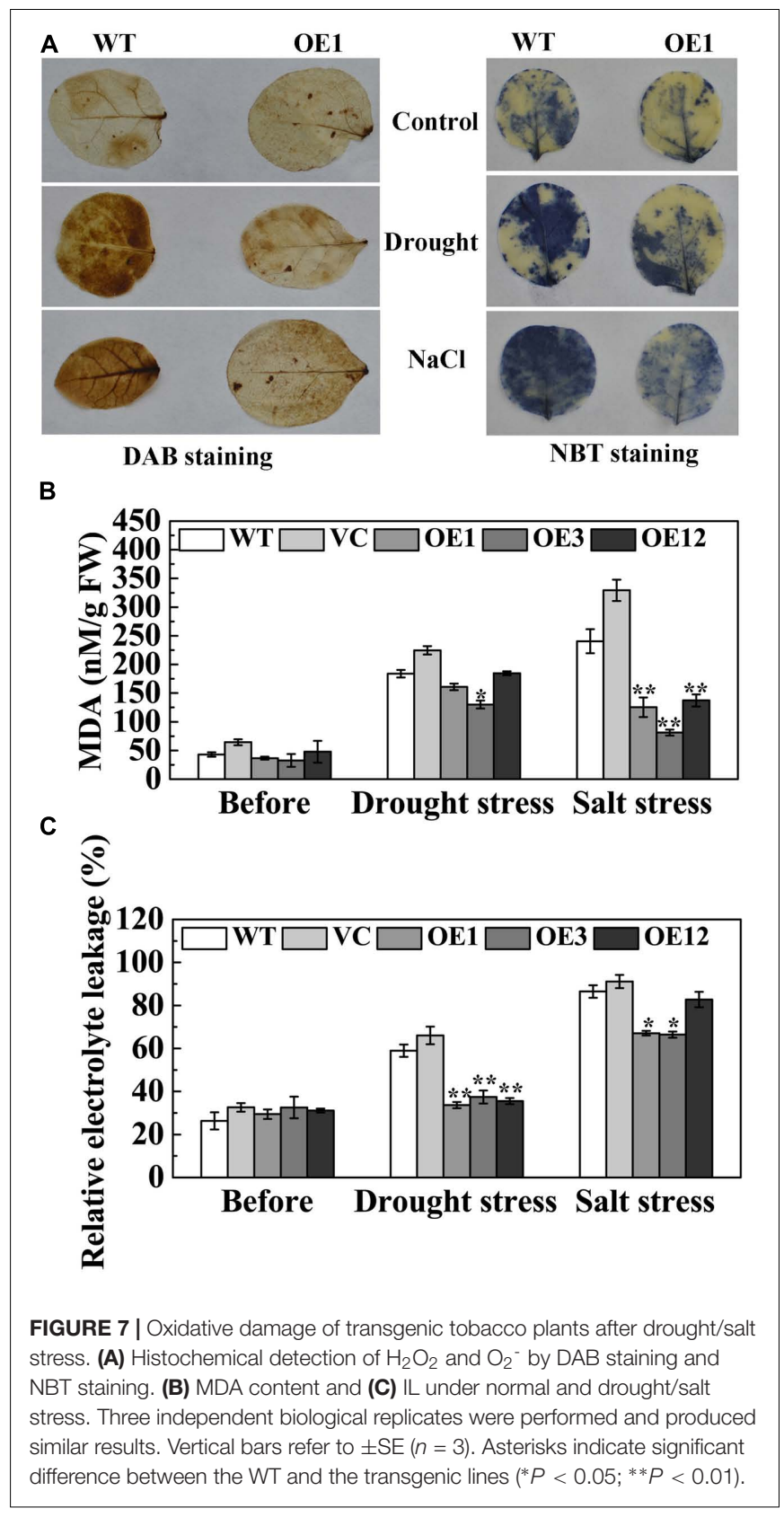

MYB gene TaODORANT1 was cloned. Sequence alignment showed that TaODORANT1 had 98\% identity with protein ODORANT1 (EMT12444) from A. tauschii which has yet to be functionally characterized. Promoter element analysis suggested that TaODORANT1 may be involved in responses to abiotic stress in plants. For example, the MBS cis-element is observed in the promoter of RD29, which has been identified to be a typical desiccation-responsive gene (Yamaguchi-Shinozaki and Shinozaki, 1993). The up-regulated expression of TaODORANT1 in wheat after PEG6000, $\mathrm{NaCl}, \mathrm{ABA}$, and $\mathrm{H}_{2} \mathrm{O}_{2}$ treatments, also suggested that this gene was involved in abiotic stress in plants (Figure 3B). Tobacco plants that over-expressed TaODORANT1 had enhanced drought and salt stress tolerance
(Figures 4-6), which is similar to the function of other $M Y B$ genes in wheat (Mao et al., 2011; He et al., 2012; Qin et al., 2012; Zhang L.C. et al., 2012).

Under drought stress, stomatal closure in TaODORANT1 transgenic plants was more sensitive to dehydration and ABA treatments than that in WT plants. Moreover, TaODORANT1-overexpressing plants had higher RWC and lower water loss in leaves (Figures 5C-G). Chen et al. (2015) also observed that GbMYB5 decreased stomatal aperture and reduced water loss rate in transgenic tobacco under drought stress. A reduced rate of water loss is crucial for plant survival under drought conditions. Transpiration, the process by which water is transported to leaves and released vapor through stomatal pores, is the principal pathway leading to water loss. Acting as a signal molecule, ABA perceives stresses and triggers changes in guard cells that stimulate stomatal closure, thus reducing transpiration (Raghavendra et al., 2010). These results agreed with previous reports for TaMYB3R1, AtMYB44, and AtMYB60, all of which have been showed to decrease the stomatal aperture in response to drought/osmotic stress (Cominelli et al., 2005; Jung et al., 2008; Cai et al., 2015). These results suggested that TaODORANT1 might be involved in ABA-mediated stomatal closure in adaptation to adverse environmental conditions.

Under salt stress, a higher $\mathrm{Na}^{+}$concentration disrupts ion homeostasis, which contributes to $\mathrm{Na}^{+}$toxicity and disrupts $\mathrm{K}^{+}$ homeostasis in the cytosol. $\mathrm{Na}^{+}$concentration in the cytoplasm is maintained by restricting $\mathrm{Na}^{+}$uptake, promoting $\mathrm{Na}^{+}$extrusion across the plasma membrane, and sequestering $\mathrm{Na}^{+}$in vacuoles under a high-salt environment (Bartels and Sunkar, 2005). The gene SOS1 encodes a plasma membrane $\mathrm{Na}^{+} / \mathrm{H}^{+}$antiporter that transports $\mathrm{Na}^{+}$out of cells and partitions $\mathrm{Na}^{+}$between plant organs. AtSOS1 overexpression improves salt tolerance in tobacco by maintaining higher $\mathrm{K}^{+} / \mathrm{Na}^{+}$ratios (Yue et al., 2012). The SISOS1 antiporter not only maintains ion homeostasis, but also partitions $\mathrm{Na}^{+}$to stems to reduce $\mathrm{Na}^{+}$contents in photosynthetic tissues (Olias et al., 2009). Coincidentally, the SbSOS1 antiporter partitions $\mathrm{Na}^{+}$to stem and xylem tissues to reduce $\mathrm{Na}^{+}$in leaves and roots, thereby enhancing the salt stress tolerance of tobacco plants (Yadav et al., 2012). The gene NHX2 encodes a $\mathrm{K}^{+}$and $\mathrm{Na}^{+} / \mathrm{H}^{+}$antiporter, which is localized in the tonoplast. AtNHX2, as a salt tolerance determinant, functions in compartmentalizing $\mathrm{Na}^{+}$in the vacuole (Yokoi et al., 2002). In the present study, compared with WT, TaODORANT1-overexpressing lines exhibited elevated NtSOS1 and NtNHX2 expression levels and increased $\mathrm{K}^{+} / \mathrm{Na}^{+}$ratio in leaves after salt stress (Figures $6 \mathrm{C}-\mathrm{F}$ ). These results demonstrated that TaODORANT1 increased the $\mathrm{K}^{+} / \mathrm{Na}^{+}$ratio in leaves by regulating ion transporters to enhance tolerance to salt stress.

Drought and salt stresses can lead to redundant ROS accumulation, resulting in severe oxidative damage in plants. ROS accumulation causes peroxidation of membrane lipids, which produces a mass of degradation products, such as MDA (Moore and Roberts, 1998). ROS accumulation is also responsible for severe electrolyte leakage. Demidchik et al. (2014) indicated that ROS-activated outwardly rectifying $\mathrm{K}^{+}$channels resulted in $\mathrm{K}^{+}$release, a major component of electrolyte leakage, from plant 


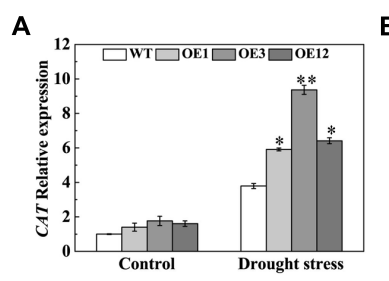

E

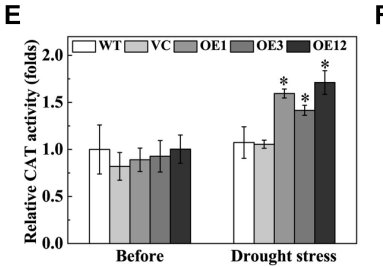

I

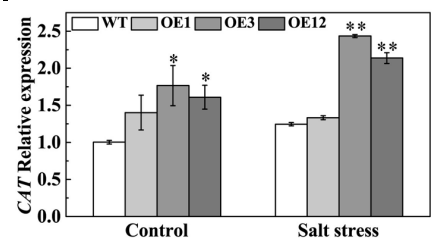

$\mathbf{F}$
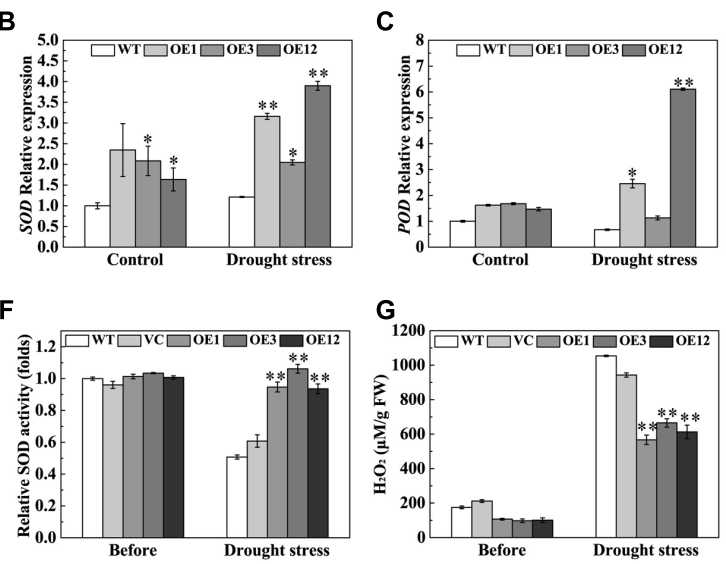

G

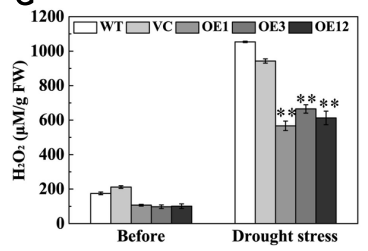

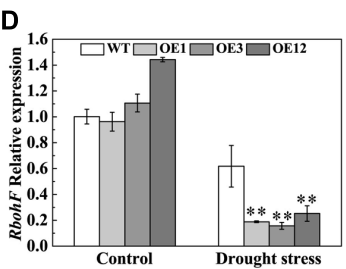

H

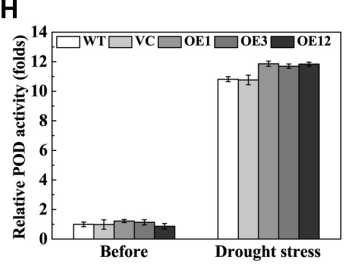

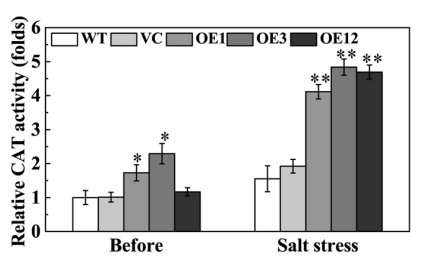

K

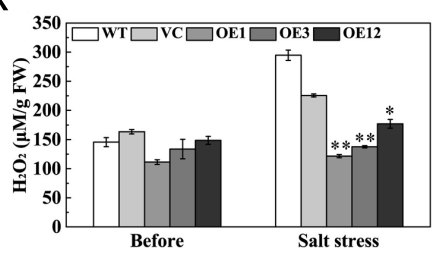

FIGURE 8 | Overexpression of TaODORANT1 in tobacco elevated antioxidant enzymes gene expression and activities under drought/salt stress. (A-D) Expression levels of CAT, SOD, POD, and RbohF in tobacco seedlings after $300 \mathrm{mM}$ mannitol treatment for 7 days. (E-H) $\mathrm{H}_{2} \mathrm{O}_{2}$ content and activities of CAT, SOD, and POD in plant leaves after drought treatment. (I) Expression levels of CAT in tobacco seedlings after $150 \mathrm{mM} \mathrm{NaCl}$ treatment for 7 days. (J,K) $\mathrm{CAT}$ activity and $\mathrm{H}_{2} \mathrm{O}_{2}$ content in plant leaves after $\mathrm{NaCl}$ treatment. Three independent biological replicates were performed and produced similar results. Vertical bars refer to $\pm \mathrm{SE}(n=3)$.

Asterisks indicate significant difference between the WT and the transgenic lines $\left({ }^{*} P<0.05\right.$; $\left.{ }^{*} P<0.01\right)$.
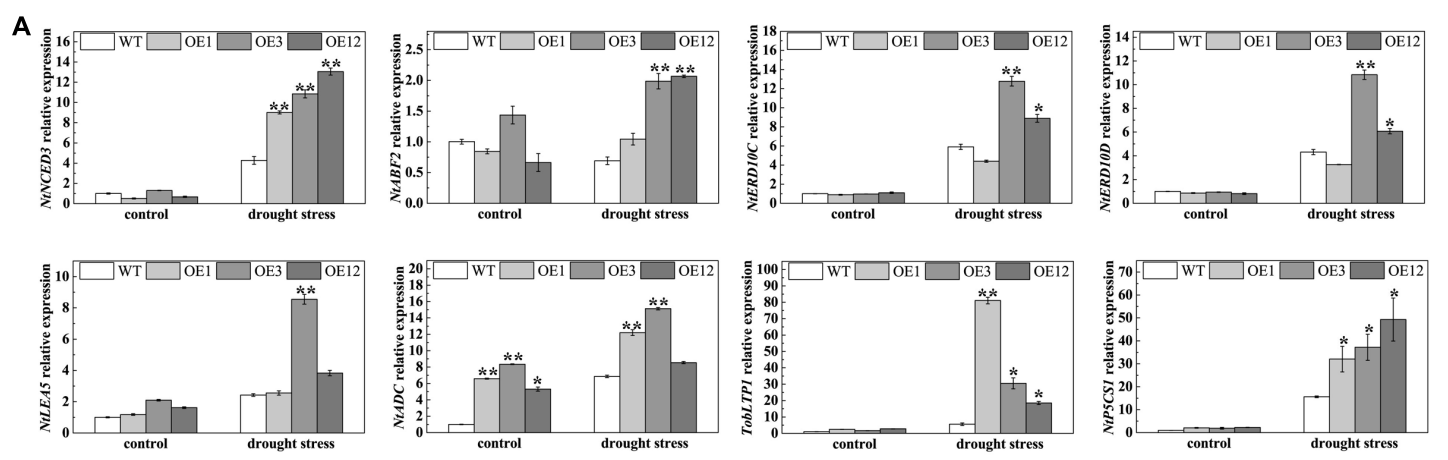

B
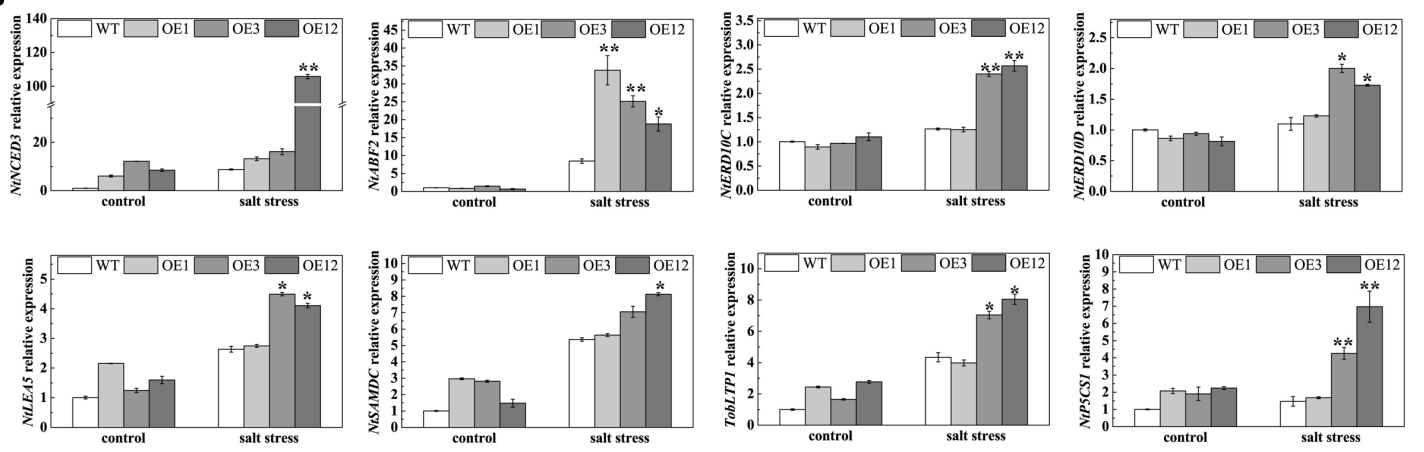

FIGURE 9 | Expression analysis of stress-related genes. (A,B) Expression levels of stress-related genes in tobacco seedlings after treatment on 1/2 MS media with $300 \mathrm{mM}$ mannitol or $150 \mathrm{mM} \mathrm{NaCl}$ for 1 week. Three independent biological replicates were performed and produced similar results. Vertical bars refer to $\pm \mathrm{SE}$ $(n=3)$. Asterisks indicate significant difference between the WT and the transgenic lines $\left({ }^{*} P<0.05 ;{ }^{* *} P<0.01\right)$. 
cells. To scavenge ROS accumulation, plants form an enzymatic system that consists of ROS-scavenging enzymes, such as SOD, CAT, and POD. Under drought stress, the expression levels and the enzyme activities of SOD and CAT were higher in transgenic plants than those in control plants. Meanwhile, the expression levels of RbohF, which encodes a ROS producer, decreased in OE plants (Figures 8A-F). Under salt stress, both expression level and enzyme activity of CAT were higher in transgenic plants (Figures 8I,J). However, POD activity under drought stress as well as POD and SOD activities under salt stress were not significantly increased compared with the WT (Figure $\mathbf{8 H}$ and Supplementary Figure S2). The results indicated that the antioxidative system is complicated. Antioxidant capacity is very much dependent on the severity and type of stress. Under different stress treatments, various plants may favor one mechanism to preferentially scavenge redundant ROS (Dat et al., 2000). The alleviative oxidative damage in transgenic tobacco plants was corroborated by decreased IL, and $\mathrm{H}_{2} \mathrm{O}_{2}$ and MDA contents (Figures $\mathbf{7}, \mathbf{8 G}, \mathbf{K}$ ). These results demonstrated that TaODORANT1 activated the ROS-scavenging system to relieve oxidative damage and enhance drought and salt tolerance.

To further understand the mechanism of enhanced stress tolerance in TaODORANT1-overexpressing transgenic plants, the transcript levels of several stress-related genes were examined. NCED encodes the rate-limiting enzyme of ABA biosynthesis and accumulation (Qin and Zeevaart, 1999). ABF2 encodes a bZIP TF that specifically binds to the ABRE cis-element; this $\mathrm{TF}$ is involved in the ABA signal pathway, which responds to abiotic stress (Yoshida et al., 2010). LEA5 and ERD10 $(C / D)$ encode group 2 and group 5 LEA proteins, respectively; these proteins maintain the structural stability of proteins and membranes (Amara et al., 2012). The lipid-transfer protein gene LTP1 is induced by ABA, cold, drought, and salt stress (Hu et al., 2013). The enzyme genes NtP5CS1, NtSAMDC, and $N A D C$ participate in biosynthesis of the osmoprotectants proline and polyamine, which function in resisting adverse environments by adjusting osmotic balance and protecting plasma membrane integrity (Bartels and Sunkar, 2005). All the above-mentioned genes were up-regulated in TaODORANT1overexpressing tobacco plants under drought and salt stresses (Figure 9). These results demonstrated that TaODORANT1

\section{REFERENCES}

Agarwal, M., Hao, Y. J., Kapoor, A., Dong, C. H., Fujii, H., Zheng, X. W., et al. (2006). A R2R3 type MYB transcription factor is involved in the cold regulation of $\mathrm{CBF}$ genes and in acquired freezing tolerance. J. Biol. Chem. 281, 37636-37645. doi: 10.1074/jbc.M605895200

Amara, I., Odena, A., Oliveira, E., Moreno, A., Masmoudi, K., Pages, M., et al. (2012). Insights into Maize LEA proteins: from proteomics to functional approaches. Plant Cell Physiol. 53, 312-329. doi: 10.1093/pcp/pcr183

Arnon, D. I. (1949). Copper enzymes in isolated chloroplasts. Polyphenoloxidase in beta vulgaris. Plant Physiol. 24, 1-15.

Bartels, D., and Sunkar, R. (2005). Drought and salt tolerance in plants. Crit. Rev. Plant. Sci. 24, 23-58. doi: 10.1080/07352680590910410

Cai, H. S., Tian, S., Dong, H. S., and Guo, C. H. (2015). Pleiotropic effects of TaMYB3R1 on plant development and response to osmotic stress in transgenic Arabidopsis. Gene 558, 227-234. doi: 10.1016/j.gene.2014. 12.066 improves the transcription of stress-related genes in response to drought and salt stresses. The result agreed with previous studies that the stress tolerance of transgenic plants was enhanced by up-regulating the expression of stress related genes ( $\mathrm{Hu}$ et al., 2013; Wang et al., 2015).

In summary, TaODORANT1 is a MYB transcriptional activator that is induced by PEG6000, $\mathrm{NaCl}, \mathrm{ABA}$, and $\mathrm{H}_{2} \mathrm{O}_{2}$ in wheat. TaODORANT1 overexpression in tobacco enhances drought and salt tolerance by increasing RWC and decreasing water loss, as well as reducing $\mathrm{H}_{2} \mathrm{O}_{2}, \mathrm{MDA}$, and $\mathrm{Na}^{+}$accumulation. Moreover, TaODORANT1 overexpression improves the activity of antioxidant enzymes and the expression of stress-related genes. Future study of the direct downstream gene or protein targets of TaODORANT1 will contribute to further elucidation of the mechanisms of TaODORANT1mediated stress tolerance.

\section{AUTHOR CONTRIBUTIONS}

GH, GY, JC, and QW designed the experiments and wrote the paper. QW performed all experiments and analyzed the data. QL helped to perform genetic transformation and partial data analysis. RW, FZ, YH, YZ, DQ, and KL participated in physiological assays and gene expression assays. All authors read and approved the manuscript.

\section{FUNDING}

The work was supported by National Genetically Modified New Varieties of Major Projects of China (2016ZX08010004004), and the National Natural Science Foundation of China (No. 31570261).

\section{SUPPLEMENTARY MATERIAL}

The Supplementary Material for this article can be found online at: http://journal.frontiersin.org/article/10.3389/fpls.2017.01374/ full\#supplementary-material

Chen, L. G., Song, Y., Li, S. J., Zhang, L. P., Zou, C. S., and Yu, D. Q. (2012). The role of WRKY transcription factors in plant abiotic stresses. Biochim. Biophys. Acta 1819, 120-128. doi: 10.1016/j.bbagrm.2011.09.002

Chen, T. Z., Li, W. J., Hu, X. H., Guo, J. R., Liu, A. M., and Zhang, B. L. (2015). A cotton MYB transcription factor, GbMYB5, is positively involved in plant adaptive response to drought stress. Plant Cell Physiol. 56, 917-929. doi: $10.1093 / \mathrm{pcp} / \mathrm{pcv019}$

Chen, Y., Chen, Z. L., Kang, J. Q., Kang, D. M., Gu, H. Y., and Qin, G. J. (2013). AtMYB14 regulates cold tolerance in arabidopsis. Plant Mol. Biol. Rep. 31, 87-97. doi: 10.1007/s11105-012-0481-z

Choulet, F., Alberti, A., Theil, S., Glover, N., Barbe, V., Daron, J., et al. (2014). Structural and functional partitioning of bread wheat chromosome 3B. Science 345:1249721. doi: 10.1126/Science.1249721

Cominelli, E., Galbiati, M., Vavasseur, A., Conti, L., Sala, T., Vuylsteke, M., et al. (2005). A guard-cell-specific MYB transcription factor regulates stomatal movements and plant drought tolerance. Curr. Biol. 15, 1196-1200. doi: 10.1016/j.cub.2005.05.048 
Cui, M. H., Yoo, K. S., Hyoung, S., Nguyen, H. T. K., Kim, Y. Y., Kim, H. J., et al. (2013). An Arabidopsis R2R3-MYB transcription factor, AtMYB20, negatively regulates type $2 \mathrm{C}$ serine/threonine protein phosphatases to enhance salt tolerance. FEBS Lett. 587, 1773-1778. doi: 10.1016/j.febslet.2013.04.028

Dai, X. Y., Xu, Y. Y., Ma, Q. B., Xu, W. Y., Wang, T., Xue, Y. B., et al. (2007). Overexpression of an R1R2R3 MYB gene, OsMYB3R-2, increases tolerance to freezing, drought, and salt stress in transgenic Arabidopsis. Plant Physiol. 143, 1739-1751. doi: 10.1104/pp. 106.094532

Dat, J., Vandenabeele, S., Vranova, E., Van Montagu, M., Inze, D., and Van Breusegem, F. (2000). Dual action of the active oxygen species during plant stress responses. Cell. Mol. Life Sci. 57, 779-795. doi: 10.1007/s000180050041

Demidchik, V., Straltsova, D., Medvedev, S. S., Pozhvanov, G. A., Sokolik, A., and Yurin, V. (2014). Stress-induced electrolyte leakage: the role of K+-permeable channels and involvement in programmed cell death and metabolic adjustment. J. Exp. Bot. 65, 1259-1270. doi: 10.1093/jxb/eru004

Dubos, C., Stracke, R., Grotewold, E., Weisshaar, B., Martin, C., and Lepiniec, L. (2010). MYB transcription factors in Arabidopsis. Trends Plant Sci. 15, 573-581. doi: $10.1016 /$ j.tplants.2010.06.005

Gao, S., Zhang, Y. L., Yang, L., Song, J. B., and Yang, Z. M. (2014). AtMYB20 is negatively involved in plant adaptive response to drought stress. Plant Soil 376, 433-443. doi: 10.1007/s11104-013-1992-6

Guo, L., Yang, H. B., Zhang, X. Y., and Yang, S. H. (2013). Lipid transfer protein 3 as a target of MYB96 mediates freezing and drought stress in Arabidopsis. J. Exp. Bot. 64, 1755-1767. doi: 10.1093/jxb/ert040

He, Y., Li, W., Lv, J., Jia, Y., Wang, M., and Xia, G. (2012). Ectopic expression of a wheat MYB transcription factor gene, TaMYB73, improves salinity stress tolerance in Arabidopsis thaliana. J. Exp. Bot. 63, 1511-1522. doi: 10.1093/jxb/ err389

Horsch, R. B., Fry, J. E., Hoffmann, N. L., Eichholtz, D., Rogers, S. C., and Fraley, R. T. (1985). A simple and general method for transferring genes into plants. Science 227, 1229-1231. doi: 10.1126/science.227.4691.1229

Hu, W., Huang, C., Deng, X. M., Zhou, S. Y., Chen, L. H., Li, Y., et al. (2013). TaASR 1, a transcription factor gene in wheat, confers drought stress tolerance in transgenic tobacco. Plant Cell Environ. 36, 1449-1464. doi: 10.1111/pce.12074

Huang, P., Chen, H., Mu, R., Yuan, X., Zhang, H. S., and Huang, J. (2015). OsMYB511 encodes a MYB domain transcription activator early regulated by abiotic stress in rice. Genet Mol. Res. 14, 9506-9517. doi: 10.4238/2015.August. 14.14

Huang, Q., Wang, Y., Li, B., Chang, J. L., Chen, M. J., Li, K. X., et al. (2015). TaNAC29, a NAC transcription factor from wheat, enhances salt and drought tolerance in transgenic Arabidopsis. BMC Plant Biol. 15:268. doi: 10.1186/ S12870-015-0644-9

Jia, J. Z., Zhao, S. C., Kong, X. Y., Li, Y. R., Zhao, G. Y., He, W. M., et al. (2013). Aegilops tauschii draft genome sequence reveals a gene repertoire for wheat adaptation. Nature 496, 91-95. doi: 10.1038/nature12028

Jung, C., Seo, J. S., Han, S. W., Koo, Y. J., Kim, C. H., Song, S. I., et al. (2008). Overexpression of AtMYB44 enhances stomatal closure to confer abiotic stress tolerance in transgenic Arabidopsis. Plant Physiol. 146, 623-635. doi: 10.1104/ pp.107.110981

Katiyar, A., Smita, S., Lenka, S. K., Rajwanshi, R., Chinnusamy, V., and Bansal, K. C. (2012). Genome-wide classification and expression analysis of MYB transcription factor families in rice and Arabidopsis. BMC Genomics 13:544. doi: 10.1186/1471-2164-13-544

Lee, T. G., Jang, C. S., Kim, J. Y., Kim, D. S., Park, J. H., Kim, D. Y., et al. (2006). A Myb transcription factor (TaMyb1) from wheat roots is expressed during hypoxia: roles in response to the oxygen concentration in root environment and abiotic stresses. Physiol. Plant. 129, 375-385. doi: 10.1111/j.1399-3054.2006. 00828.x

Ling, H. Q., Zhao, S. C., Liu, D. C., Wang, J. Y., Sun, H., Zhang, C., et al. (2013). Draft genome of the wheat A-genome progenitor Triticum urartu. Nature 496, 87-90. doi: 10.1038/nature11997

Liu, H. X., Zhou, X. Y., Dong, N., Liu, X., Zhang, H. Y., and Zhang, Z. Y. (2011). Expression of a wheat MYB gene in transgenic tobacco enhances resistance to Ralstonia solanacearum, and to drought and salt stresses. Funct. Integr. Genomics 11, 431-443. doi: 10.1007/s10142-011-0228-1

Livak, K. J., and Schmittgen, T. D. (2001). Analysis of relative gene expression data using real-time quantitative PCR and the $2^{-\Delta \Delta C_{\mathrm{T}}}$ method. Methods 25, 402-408. doi: 10.1006/meth.2001.1262
Mao, X., Jia, D., Li, A., Zhang, H., Tian, S., Zhang, X., et al. (2011). Transgenic expression of TaMYB2A confers enhanced tolerance to multiple abiotic stresses in Arabidopsis. Funct. Integr. Genomics 11, 445-465. doi: 10.1007/s10142-0110218-3

Mayer, K. F. X., Rogers, J., Dolezel, J., Pozniak, C., Eversole, K., Feuillet, C., et al. (2014). A chromosome-based draft sequence of the hexaploid bread wheat (Triticum aestivum) genome. Science 345, 1251788. doi: 10.1126/Science. 1251788

Meng, X., Yin, B., Feng, H. L., Zhang, S., Liang, X. Q., and Meng, Q. W. (2014). Overexpression of R2R3-MYB gene leads to accumulation of anthocyanin and enhanced resistance to chilling and oxidative stress. Biol. Plant. 58, 121-130. doi: 10.1007/s10535-013-0376-3

Moore, K., and Roberts, L. J. II (1998). Measurement of lipid peroxidation. Free Radic. Res. 28, 659-671.

Olias, R., Eljakaoui, Z., Li, J., De Morales, P. A., Marin-Manzano, M. C., Pardo, J. M., et al. (2009). The plasma membrane $\mathrm{Na}+/ \mathrm{H}+$ antiporter $S O S 1$ is essential for salt tolerance in tomato and affects the partitioning of $\mathrm{Na}+$ between plant organs. Plant Cell Environ. 32, 904-916. doi: 10.1111/j.1365-3040.2009.01971.x

Pasquali, G., Biricolti, S., Locatelli, F., Baldoni, E., and Mattana, M. (2008). Osmyb4 expression improves adaptive responses to drought and cold stress in transgenic apples. Plant Cell Rep. 27, 1677-1686. doi: 10.1007/s00299-008-0587-9

Paz-Ares, J., Ghosal, D., Wienand, U., Peterson, P. A., and Saedler, H. (1987). The regulatory $\mathrm{cl}$ locus of Zea mays encodes a protein with homology to myb protooncogene products and with structural similarities to transcriptional activators. EMBO J. 6, 3553-3558.

Polle, A. (2001). Dissecting the superoxide dismutase-ascorbate-glutathionepathway in chloroplasts by metabolic modeling. Computer simulations as a step towards flux analysis. Plant Physiol. 126, 445-462.

Qin, F., Shinozaki, K., and Yamaguchi-Shinozaki, K. (2011). Achievements and challenges in understanding plant abiotic stress responses and tolerance. Plant Cell Physiol. 52, 1569-1582. doi: 10.1093/pcp/pcr106

Qin, X., and Zeevaart, J. A. (1999). The 9-cis-epoxycarotenoid cleavage reaction is the key regulatory step of abscisic acid biosynthesis in water-stressed bean. Proc. Natl. Acad. Sci. U.S.A. 96, 15354-15361.

Qin, Y., Wang, M., Tian, Y., He, W., Han, L., and Xia, G. (2012). Over-expression of TaMYB33 encoding a novel wheat MYB transcription factor increases salt and drought tolerance in Arabidopsis. Mol. Biol. Rep. 39, 7183-7192. doi: 10.1007/ s11033-012-1550-y

Raghavendra, A. S., Gonugunta, V. K., Christmann, A., and Grill, E. (2010). ABA perception and signalling. Trends Plant Sci. 15, 395-401. doi: 10.1016/j.tplants. 2010.04.006

Rahaie, M., Xue, G. P., Naghavi, M. R., Alizadeh, H., and Schenk, P. M. (2010). A MYB gene from wheat (Triticum aestivum L.) is up-regulated during salt and drought stresses and differentially regulated between salt-tolerant and sensitive genotypes. Plant Cell Rep. 29, 835-844. doi: 10.1007/s00299-010-0868-y

Schroeder, J. I., Allen, G. J., Hugouvieux, V., Kwak, J. M., and Waner, D. (2001). Guard cell signal transduction. Annu. Rev. Plant Physiol. Plant Mol. Biol. 52, 627-658. doi: 10.1146/annurev.arplant.52.1.627

Seo, P. J., Xiang, F., Qiao, M., Park, J. Y., Lee, Y. N., Kim, S. G., et al. (2009). The MYB96 transcription factor mediates abscisic acid signaling during drought stress response in Arabidopsis. Plant Physiol. 151, 275-289. doi: 10.1104/pp.109. 144220

Staccy, J., and Isaac, P. G. (1994). Isolation of DNA from plants. Methods Mol. Biol. 28, 9-15. doi: 10.1385/0-89603-254-X:9

Tamura, K., Peterson, D., Peterson, N., Stecher, G., Nei, M., and Kumar, S. (2011). MEGA5: molecular evolutionary genetics analysis using maximum likelihood, evolutionary distance, and maximum parsimony methods. Mol. Biol. Evol. 28, 2731-2739. doi: 10.1093/molbev/msr121

Wang, X., Zeng, J., Li, Y., Rong, X., Sun, J., Sun, T., et al. (2015). Expression of TaWRKY44, a wheat WRKY gene, in transgenic tobacco confers multiple abiotic stress tolerances. Front. Plant Sci. 6:615. doi: 10.3389/fpls.2015. 00615

Xiong, H. Y., Li, J. J., Liu, P. L., Duan, J. Z., Zhao, Y., Guo, X., et al. (2014). Overexpression of OsMYB48-1, a novel MYB-relate transcription factor, enhances drought and salinity tolerance in rice. PLoS ONE 9:e92913. doi: 10.1371/journal.pone.0092913

Yadav, N. S., Shukla, P. S., Jha, A., Agarwal, P. K., and Jha, B. (2012). The SbSOS1 gene from the extreme halophyte Salicornia brachiata enhances $\mathrm{Na}+$ loading in 
xylem and confers salt tolerance in transgenic tobacco. BMC Plant Biol. 12:188. doi: 10.1186/1471-2229-12-188

Yamaguchi-Shinozaki, K., and Shinozaki, K. (1993). Characterization of the expression of a desiccation-responsive $r d 29$ gene of Arabidopsis thaliana and analysis of its promoter in transgenic plants. Mol. Gen. Genet. 236, 331-340.

Yan, H. R., Jia, H. H., Chen, X. B., Hao, L. L., An, H. L., and Guo, X. Q. (2014). The cotton WRKY transcription factor GhWRKY17 functions in ddrought and salt stress in transgenic Nicotiana benthamiana through ABA signaling and the modulation of reactive oxygen species production. Plant Cell Physiol. 55, 2060-2076. doi: 10.1093/pcp/pcu133

Yang, A., Dai, X. Y., and Zhang, W. H. (2012). A R2R3-type MYB gene, OsMYB2, is involved in salt, cold, and dehydration tolerance in rice. J. Exp. Bot. 63, 2541-2556. doi: 10.1093/jxb/err431

Yokoi, S., Quintero, F. J., Cubero, B., Ruiz, M. T., Bressan, R. A., Hasegawa, P. M., et al. (2002). Differential expression and function of Arabidopsis thaliana $\mathrm{NHX} \mathrm{Na}+/ \mathrm{H}+$ antiporters in the salt stress response. Plant J. 30, 529-539. doi: 10.1046/j.1365-313X.2002.01309.x

Yoshida, T., Fujita, Y., Sayama, H., Kidokoro, S., Maruyama, K., Mizoi, J., et al. (2010). AREB1, AREB2, and ABF3 are master transcription factors that cooperatively regulate ABRE-dependent ABA signaling involved in drought stress tolerance and require ABA for full activation. Plant J. 61, 672-685. doi: 10.1111/j.1365-313X.2009.04092.x

Yue, Y., Zhang, M., Zhang, J., Duan, L., and Li, Z. (2012). SOS1 gene overexpression increased salt tolerance in transgenic tobacco by maintaining a higher $\mathrm{K}(+) / \mathrm{Na}(+)$ ratio. J. Plant Physiol. 169, 255-261. doi: 10.1016/j.jplph.2011. 10.007

Zhang, L., Liu, G., Zhao, G., Xia, C., Jia, J., Liu, X., et al. (2014). Characterization of a wheat R2R3-MYB transcription factor gene, TaMYB19, involved in enhanced abiotic stresses in Arabidopsis. Plant Cell Physiol. 55, 1802-1812. doi: 10.1093/ pcp/pcu109

Zhang, L. C., Zhao, G. Y., Xia, C., Jia, J. Z., Liu, X., and Kong, X. Y. (2012). Overexpression of a wheat MYB transcription factor gene, TaMYB56-B, enhances tolerances to freezing and salt stresses in transgenic Arabidopsis. Gene 505, 100-107. doi: 10.1016/j.gene.2012. 05.033

Zhang, Z. Y., Liu, X., Wang, X. D., Zhou, M. P., Zhou, X. Y., Ye, X. G., et al. (2012). An R2R3 MYB transcription factor in wheat, TaPIMP1, mediates host resistance to Bipolaris sorokiniana and drought stresses through regulation of defenseand stress-related genes. New Phytol. 196, 1155-1170. doi: 10.1111/j.1469-8137. 2012.04353.x

Zhu, N., Cheng, S., Liu, X., Du, H., Dai, M., Zhou, D. X., et al. (2015). The R2R3type MYB gene OsMYB91 has a function in coordinating plant growth and salt stress tolerance in rice. Plant Sci. 236, 146-156. doi: 10.1016/j.plantsci.2015. 03.023

Conflict of Interest Statement: The authors declare that the research was conducted in the absence of any commercial or financial relationships that could be construed as a potential conflict of interest.

Copyright (c) 2017 Wei, Luo, Wang, Zhang, He, Zhang, Qiu, Li, Chang, Yang and He. This is an open-access article distributed under the terms of the Creative Commons Attribution License (CC BY). The use, distribution or reproduction in other forums is permitted, provided the original author(s) or licensor are credited and that the original publication in this journal is cited, in accordance with accepted academic practice. No use, distribution or reproduction is permitted which does not comply with these terms. 